ARTICLE

DOI: $10.1038 / s 41467-017-01521-4$

\title{
Iced photochemical reduction to synthesize atomically dispersed metals by suppressing nanocrystal growth
}

Hehe Wei ${ }^{1}$, Kai Huang ${ }^{1}$, Da Wang ${ }^{2}{ }^{2}$, Ruoyu Zhang ${ }^{1}$, Binghui $\mathrm{Ge}^{3}$, Jingyuan Ma ${ }^{4}$, Bo Wen², Shuai Zhang ${ }^{5}$, Qunyang $\mathrm{Li}^{5}$, Ming Lei ${ }^{6}$, Cheng Zhang ${ }^{7}$, Joshua Irawan ${ }^{8}$, Li-Min Liu ${ }^{9,2} \&$ Hui Wu ${ }^{1}$

Photochemical solution-phase reactions have been widely applied for the syntheses of nanocrystals. In particular, tuning of the nucleation and growth of solids has been a major area of focus. Here we demonstrate a facile approach to generate atomically dispersed platinum via photochemical reduction of frozen chloroplatinic acid solution using ultraviolet light. Using this iced-photochemical reduction, the aggregation of atoms is prevented, and single atoms are successfully stabilized. The platinum atoms are deposited on various substrates, including mesoporous carbon, graphene, carbon nanotubes, titanium dioxide nanoparticles, and zinc oxide nanowires. The atomically dispersed platinum on mesoporous carbon exhibits efficient catalytic activity for the electrochemical hydrogen evolution reaction, with an overpotential of only $65 \mathrm{mV}$ at a current density of $100 \mathrm{~mA} \mathrm{~cm}^{-2}$ and long-time durability $(>10 \mathrm{~h})$, superior to state-of-the-art platinum/carbon. This iced-photochemical reduction may be extended to other single atoms, for example gold and silver, as demonstrated in this study.

\footnotetext{
${ }^{1}$ State Key Laboratory of New Ceramics and Fine Processing, School of Materials Science and Engineering, Tsinghua University, 100084 Beijing, China.

2 Beijing Computational Science Research Center, 100193 Beijing, China. ${ }^{3}$ Beijing National Laboratory for Condensed Matter Physics, Institute of Physics, Chinese Academy of Sciences, 100190 Beijing, China. ${ }^{4}$ Shanghai Synchrotron Radiation Facility, Shanghai Institute of Applied Physics, Chinese Academy of Sciences, 201204 Shanghai, China. ${ }^{5}$ AML, CNMM, Department of Engineering Mechanics, State Key Laboratory of Tribology, Tsinghua University, 100084 Beijing, China. ${ }^{6}$ State Key Laboratory of Information Photonics and Optical Communications and School of Science, Beijing University of Posts and Telecommunications, 100876 Beijing, China. ${ }^{7}$ School of Materials Science and Engineering, Shanghai Institute of Technology, 201418 Shanghai, China. ${ }^{8}$ School of Material Science and Engineering, University of New South Wales, Sydney, NSW 2052, Australia. ${ }^{9}$ School of Physics and Nuclear Energy Engineering, Beihang University, Beijing 100191, China. Hehe Wei, Kai Huang, Da Wang and Ruoyu Zhang contributed equally to this work. Correspondence and requests for materials should be addressed to B.G. (email: bhge@iphy.ac.cn) or to L.-M.L. (email: liminliu@buaa.edu.cn)

or to H.W. (email: huiwu@tsinghua.edu.cn)
} 
A tomically dispersed metals have become an area of growing scientific interest due to their unique chemical and physical properties, as well as their applications as high performance catalysts ${ }^{1-6}$. The synthesis of metal single atoms by traditional solution chemistry methods is extremely difficult mainly because of the diffusion, aggregation and nucleation of the product atoms in the liquid phase. In fact, for over a century, in-solution chemical and photochemical reduction of metal ions has been studied as one of the most common methodologies for the synthesis of metal nanocrystals ${ }^{7-14}$. Controlling the morphology and size of these nanocrystals has relied strongly on the fundamental understanding of the nucleation and growth of solids during liquid phase reactions. In typical liquid phase chemical/photochemical reductions, it is recognized that nucleation represents the very first step that atomic or molecular species must undergo before emerging as nanocrystals; generally, a precursor is reduced or decomposed to generate atoms, followed by their evolution into nuclei and then nanocrystals ${ }^{15}, 16$. Recently, using in situ electron microscopy, Mirsaidov et al. ${ }^{17}$ demonstrated that the reduction of metal ions in an aqueous solution results in nanocrystals in three major steps: spinodal decomposition into solute-rich and solute-poor liquid phases, nucleation of amorphous nanoclusters within the metal-rich liquid phase, and crystallization of these amorphous clusters ${ }^{17}$. It has been recognized that to generate sub-nanometer clusters or even single atoms, the diffusion of atoms and the formation of metal nuclei should be prevented.

Recently, a number of strategies have been proposed for downsizing solution processed nanocrystals to sub-nanometer clusters or even single atoms. Confining the chemical/photochemical reduction process to a nanoscale space can greatly influence the nucleation of materials. Sub-nanometer clusters or single atoms can be obtained in the confined pores of zeolite ${ }^{18}$, mesoporous carbon, metal organic frameworks (MOFs), and so on, using solution synthesis routes such as the impregnation $\operatorname{method}^{19,20}$ and the organometallic complex method 21,22 . Despite significant progress, the demonstration of facile and highyield synthesis of atomically dispersed metals with high density and high stability remains challenging. It is an interesting and open question whether, we can effectively prevent the nucleation and crystal growth in solution synthesis. For example, photochemical reduction chemistry, which involves the absorption of photons and electronically excited states 23,24 , has been widely applied for the syntheses of nanomaterials 25,26 , especially for nanocrystals of noble metals, including gold, silver, platinum, palladium, and so on ${ }^{27,28}$. In this process, the nanocrystal formation and growth strongly rely on the diffusion of the ions and atoms inside the liquid environment. Considering this fact, we propose to freeze the precursor solution for the photochemical reaction. Instead of directly growing nanocrystals in the liquid phase, the ice lattice naturally confines the dispersed reactants and therefore possibly restricts the photochemical reduction products, preventing nucleus formation.

Herein, we experimentally demonstrate that atomically dispersed Pt can be effectively synthesized via iced-photochemical reduction using ultraviolet (UV) irradiation of frozen chloroplatinic acid $\left(\mathrm{H}_{2} \mathrm{PtCl}_{6}\right)$ aqueous solution. Pt single atoms $\left(\mathrm{Pt}_{1}\right)$ can be stabilized on various substrates, including amorphous carbon film, mesoporous carbon (MC), graphene, multi-walled carbon nanotubes (MWCNTs), $\mathrm{TiO}_{2}$ nanoparticles, and $\mathrm{ZnO}$ nanowires. The $\mathrm{Pt}_{1} / \mathrm{MC}$ material is an active and stable electrocatalyst for the hydrogen evolution reaction (HER), with significantly improved performance compared with state-of-the-art commercial platinum/carbon $(\mathrm{Pt} / \mathrm{C})$ catalysts.

\section{Results}

Concept and process of iced-chemistry. The iced-photochemical synthesis of $\mathrm{Pt}$ single atoms is illustrated in Fig. 1. First, $\mathrm{H}_{2} \mathrm{PtCl}_{6}$
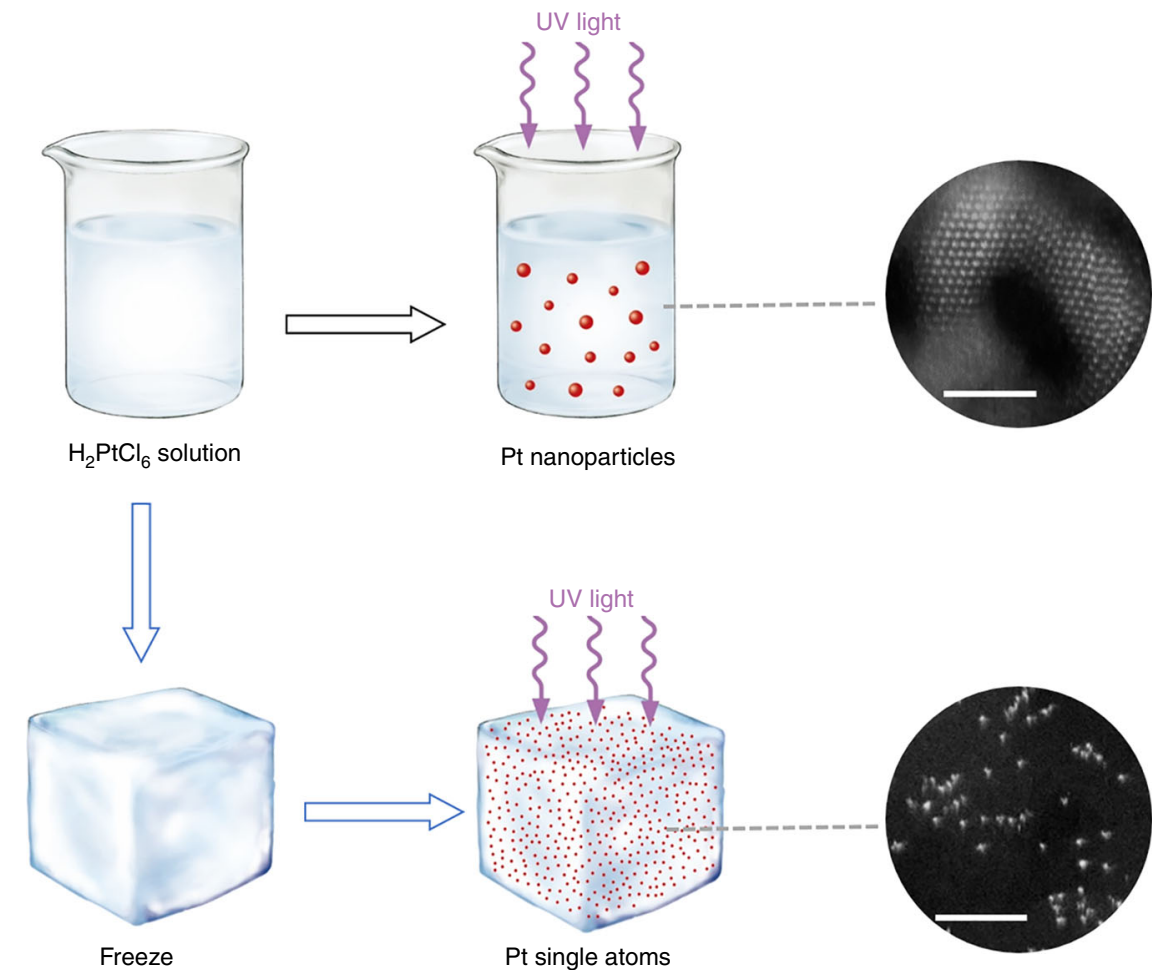

Fig. 1 Schematic illustration the iced-photochemical process. In a conventional photochemical reduction of $\mathrm{H}_{2} \mathrm{PtCl}_{6}$ aqueous solution, $\mathrm{Pt}$ nanocrystals are formed by the agglomeration and nucleation of Pt atoms (top row). Conversely, in the bottom row, we froze the $\mathrm{H}_{2} \mathrm{PtCl}_{6}$ solution before exposing it to UV irradiation and obtained Pt single atoms dispersed in ice. The ice lattice naturally confined the ions/atoms and prevented their nucleation. (scale bar, $2 \mathrm{~nm}$ ) 

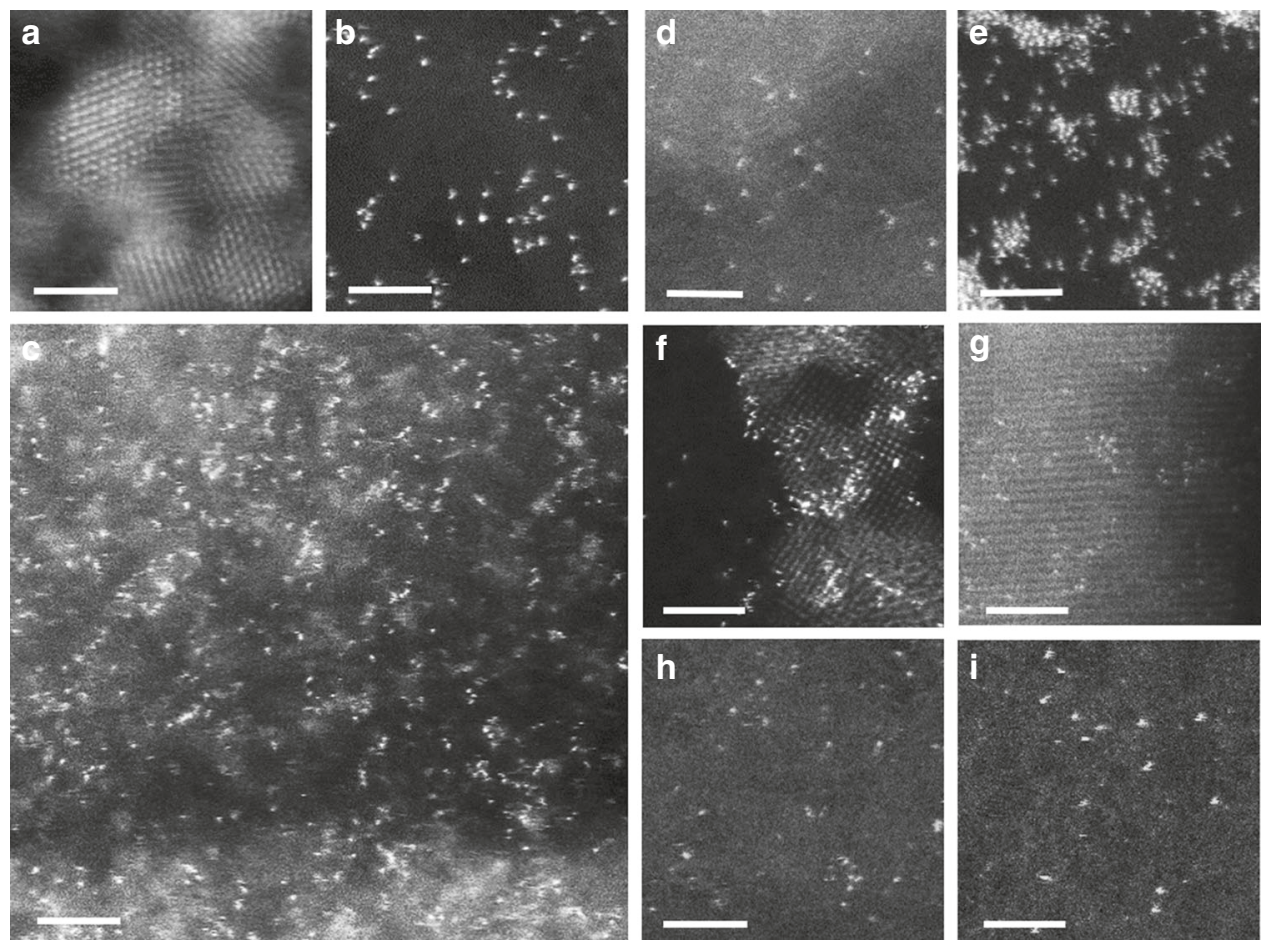

Fig. 2 HAADF-STEM images of Pt single atoms. a Pt nanocrystals with a size of $\sim 2 \mathrm{~nm}$ formed by normal photochemical reduction of $\mathrm{H}_{2} \mathrm{PtCl}_{6}$ aqueous solution. b Pt single atoms dispersed on ultrathin carbon film, with each bright dot corresponding to one individual Pt atom, with a size of $\sim 0.1 \mathrm{~nm}$. c Densely and homogeneously dispersed Pt single atoms on mesoporous carbon. $\mathbf{d} \mathrm{Pt}_{1} / \mathrm{MWCNTs}$, indicating that Pt existed completely as isolated single atoms. e Pt 1 /graphene, with concomitant Pt single atoms, nanoclusters and sub-nanometer clusters. $\mathbf{f}$ Atomically dispersed $\mathrm{Pt}$ on titanium oxide nanoparticles. $\mathbf{g}$ Pt single atoms attached on the surface of zinc oxide nanowires. $\mathbf{h ~ A g}$ and $\mathbf{i}$ Au single atoms prepared by a similar iced-photochemical route. (scale bar, $2 \mathrm{~nm}$ )

aqueous solution was rapidly frozen by dipping the container in liquid nitrogen, with a cooling rate of $40{ }^{\circ} \mathrm{C} \mathrm{min}^{-1}$ (Supplementary Fig. 1). Benefiting from the ultralow temperature of liquid nitrogen, $\mathrm{H}_{2} \mathrm{PtCl}_{6}$ aqueous solution can be frozen very quickly to yield an ice layer with a homogeneous concentration of Pt precursor (Supplementary Fig. 2). Note that all chemicals, the solution and the ice were kept in dark conditions to avoid possible $\mathrm{H}_{2} \mathrm{PtCl}_{6}$ decomposition by ambient light. Second, the frozen solution was exposed to UV irradiation on a cooling stage with a temperature of $-25^{\circ} \mathrm{C}$ for $1 \mathrm{~h}$ to ensure the photochemical reduction of $\mathrm{Pt}(\mathrm{IV})$. After the UV treatments, the frozen solution was kept overnight in dark conditions at room temperature to yield a clear aqueous solution. The solution was subsequently mixed with various dispersions containing MC, graphene, MWCNTs, $\mathrm{TiO}_{2}$ nanoparticles, and $\mathrm{ZnO}$ nanowires. After centrifuging, washing and drying the samples multiple times, the products from the iced-photochemical process were stabilized on these substrates. We characterized the products with aberrationcorrected high-angle annular dark field-scanning transmission electron microscopy (HAADF-STEM). Obviously, the traditional UV irradiation of $\mathrm{H}_{2} \mathrm{PtCl}_{6}$ aqueous solutions (as shown in the upper line of Fig. 1) produced Pt crystals with the size of a few nanometers (Fig. 2a). In comparison, the iced-photochemical reduction (lower line of Fig. 1) leads to high density areas of isolated Pt atoms (Fig. 2b, Supplementary Fig. 3). The atoms were identified as platinum, confirmed by electron energy loss spectra (EELS, Supplementary Fig. 4). No Pt nanoclusters and nanoparticles were observed, as shown in the TEM images and selected area electron diffraction (SAED) spectra (Supplementary Fig. 4d). The size distribution of Pt single atoms (Supplementary Fig. 5) showed a significant majority of isolated atoms with a size of $\sim 0.1$ nm (Supplementary Fig. 6) on ultrathin carbon film. When the concentration of $\mathrm{H}_{2} \mathrm{PtCl}_{6}$ solution reached $7.3 \mathrm{mM}$, the $\mathrm{Pt}$ single atoms partially aggregated under the same experimental conditions using the iced-photochemical reduction (Supplementary Fig. 7). The STEM images in Fig. 2c-g, Supplementary Fig. 8 display the $\mathrm{Pt}$ atoms attached on various substrates, including MC, MWCNTs, graphene, $\mathrm{TiO}_{2}$ nanoparticles, and $\mathrm{ZnO}$ nanowires. Atomically dispersed Pt can be observed on most substrates with the exception of graphene, where partially aggregated clusters have been found. The synthesized materials were further characterized with scanning tunneling microscopy (STM), X-ray absorption fine structure (XAFS), and X-ray diffraction (XRD). Figure 3a shows the typical STM images of atomically dispersed $\mathrm{Pt}$ on ultrathin carbon films. With atomic resolution, $\mathrm{Pt}$ single atoms appeared as single-peak protrusions $\sim 0.2 \mathrm{~nm}$ in diameter and $\sim 0.3 \mathrm{~nm}$ in height, in good agreement with previous literature $^{29}$. For the XAFS study, the X-ray absorption near edge structure (XANES) of Pt single atom materials $\left(\mathrm{Pt}_{1} / \mathrm{MC}\right.$ and $\mathrm{Pt}_{1} /$ $\mathrm{TiO}_{2}$ ) exhibited increased white-line intensity compared to that of the Pt foil, in accordance with previous reports ${ }^{1,5}$ (Fig. 3b). As shown in Fig. $3 \mathrm{c}$ and Supplementary Table 1, there was a notable peak 2.1 $\AA$ from the Pt-C/O contribution for $\mathrm{Pt}_{1} / \mathrm{MC}$ and $\sim 2.0 \AA$ from the Pt-O contribution for $\mathrm{Pt}_{1} / \mathrm{TiO}_{2}$ and no peak $\sim 2.76 \AA$ from the Pt-Pt contribution, confirming the isolated states of $\mathrm{Pt}$ single atoms on the mesoporous carbon. This was consistent with the direct observation of the atomic dispersion of Pt by STEM (Fig. 2). The binding energies of $\mathrm{Pt} 4 \mathrm{f}_{7 / 2}$ and $4 \mathrm{f}_{5 / 2}$ for $\mathrm{Pt}_{1} / \mathrm{MC}$ (Supplementary Fig. 9) were $72.3 \mathrm{eV}$ and $75.1 \mathrm{eV}$ (corresponding to $\mathrm{Pt}^{\delta+}$ ). The results were in agreement with previous reports ${ }^{3,5}$. $\mathrm{XRD}$ results further confirmed the absence of a Pt lattice, as shown in Supplementary Figs. 10, 11. 
a
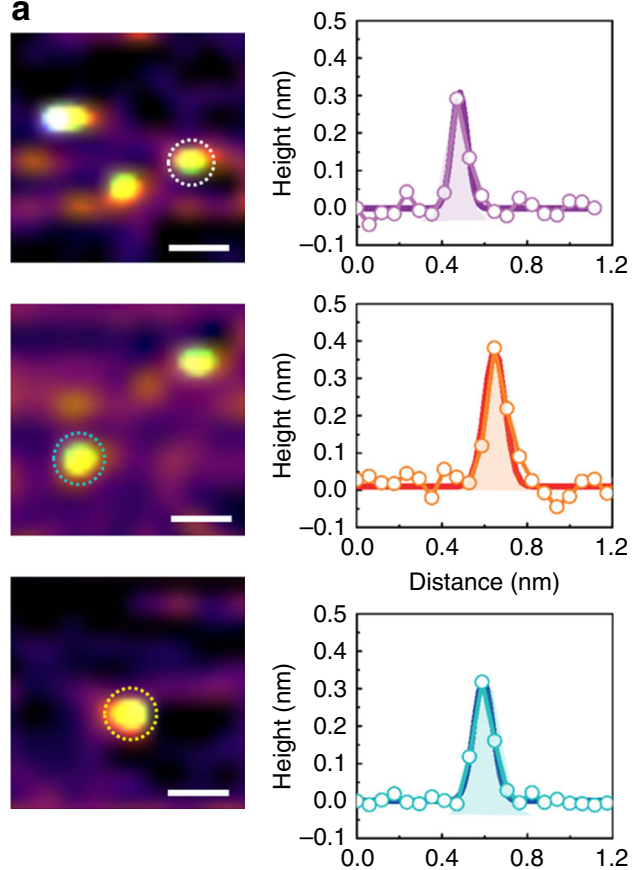

b
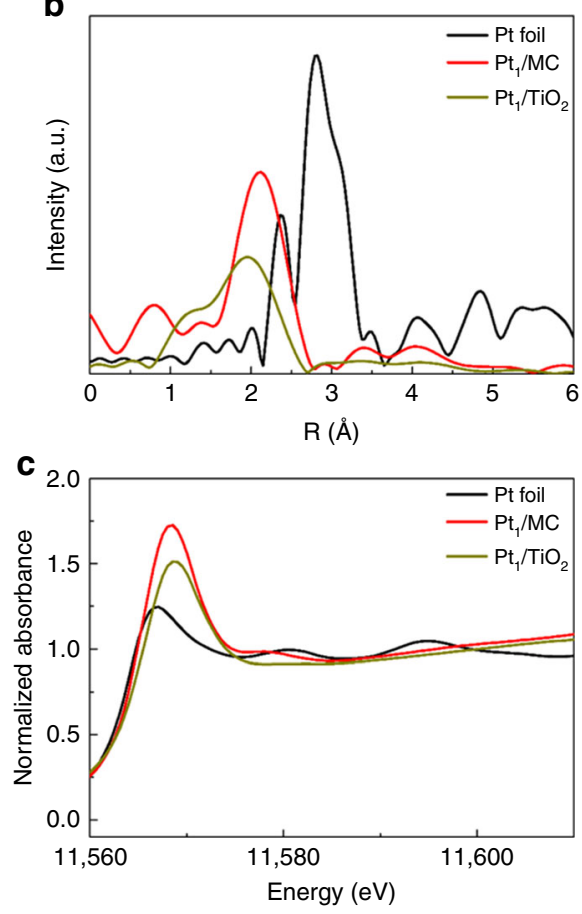

DVS

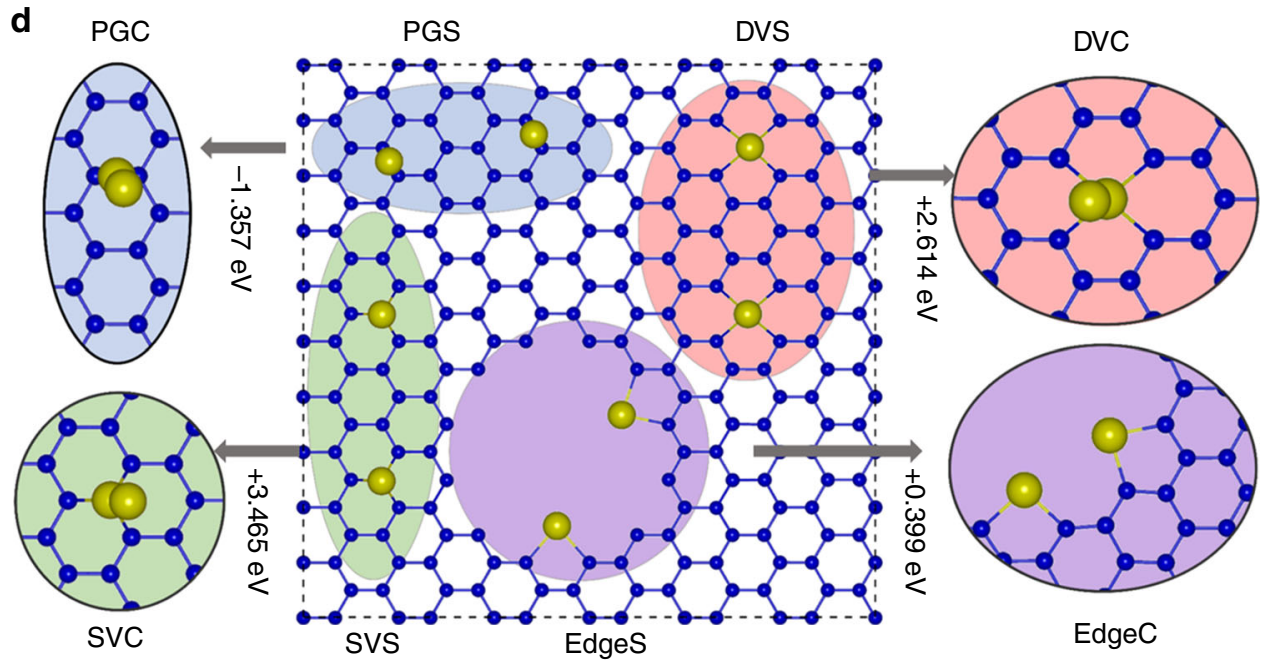

Fig. 3 Characterizations and structure of Pt single atoms. a Typical STM images of Pt single atoms dispersed on ultrathin carbon films (constant current mode). $\mathbf{b}$ EXAFS spectra of bulk Pt foil and Pt single atoms absorbed on $\mathrm{TiO}_{2}$ and $\mathrm{MC}$. c Normalized XANE structure spectra at the Pt $\mathrm{L}_{3}$-edge. $\mathbf{d}$ Structure and size distribution for Pt single atoms and Pt clusters on mesoporous carbon. The Pt single atom and clustering configurations are denoted by $\mathrm{XS}$ and $\mathrm{XC}$, respectively, where $X$ indicates the $P t$ atoms adsorbed on different defects (SVs and DVs) and edges. The yellow and blue balls represent the Pt and $C$ atoms, respectively. (scale bar, $0.2 \mathrm{~nm}$ )

Synthesis mechanism. The above experimental results demonstrated that Pt single atoms were fabricated on substrates, indicating that it is possible that the Pt single atoms could have been stabilized in water. To comprehend this, several different sizes of $\mathrm{Pt}$ cluster, such as $\mathrm{Pt}$ single atom, $\mathrm{Pt}$ dimer, and $\mathrm{Pt}_{13}$, were explored in water at both room temperature and $1000 \mathrm{~K}$ using first-principles molecular dynamics (FPMD) (Supplementary Fig. 12). For the Pt single atom system, two Pt single atoms were initially placed into water to check how they behaved in aqueous solution. The results showed that the two Pt single atoms do not form a Pt dimer in aqueous solution at $300 \mathrm{~K}$ for $100 \mathrm{ps}$ (Supplementary Fig. 12a), while the Pt single atom automatically formed the $\mathrm{H}-\mathrm{Pt}-\mathrm{OH}$ species through dissociating water molecules during FPMD at $1000 \mathrm{~K}$. To further check the oxidation state of $\mathrm{Pt}$ in $\mathrm{H}-\mathrm{Pt}-\mathrm{OH}$, the electronic structure of $\mathrm{H}-\mathrm{Pt}-\mathrm{OH}$ in water was analyzed using the form of $\mathrm{Pt}-\mathrm{H}_{2} \mathrm{O}$ as a reference (Supplementary Fig. 13, Supplementary Table 2). In the case of the Pt single atom in $\mathrm{Pt}-\mathrm{H}_{2} \mathrm{O}$, the calculated Bader charge of the $\mathrm{Pt}$ atom was $-0.094 e$; thus, no obvious charge transfer occurred between Pt and water. Meanwhile, as shown in Supplementary Table 3, only one of five Pt-d electron orbitals $\left(\mathrm{d}_{\mathrm{z}}{ }^{2}\right)$ were nearly half-filled $(1.377 e)$. In this case, the valence electron configuration of $\mathrm{Pt}$ in $\mathrm{Pt}-\mathrm{H}_{2} \mathrm{O}$ was $5 \mathrm{~d}^{9} 6 \mathrm{~s}^{1}$, suggesting the approximately neutral oxidation state for $\mathrm{Pt}$ in $\mathrm{Pt}-\mathrm{H}_{2} \mathrm{O}$. When the $\mathrm{H}-\mathrm{Pt}-\mathrm{OH}$ structure was formed, one of five Pt-d orbitals $\left(\mathrm{d}_{x}{ }^{2}-y^{2}\right)$ became nearly half-filled (1.377 e by partial charge analysis), and two Pt-d orbitals $\left(\mathrm{d}_{x y} / \mathrm{d}_{x z}\right)$ became partially filled with 1.796 and $1.809 e$. These two orbitals interacted with hydroxyl and protons, respectively. Meanwhile, the Bader charge of $\mathrm{Pt}$ in $\mathrm{H}-\mathrm{Pt}-\mathrm{OH}$ was $0.408 e$. It should be noted that the absolute oxidation state (OS) 
a

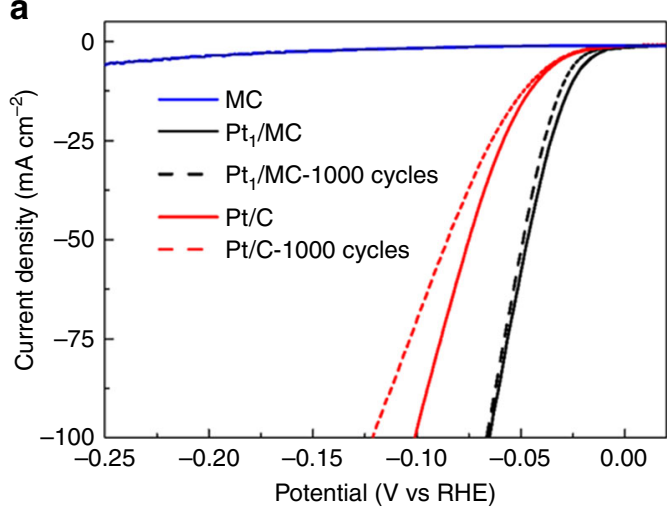

C

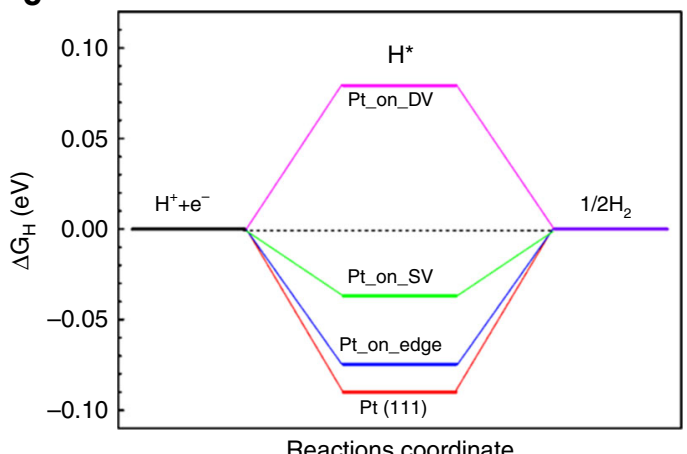

b

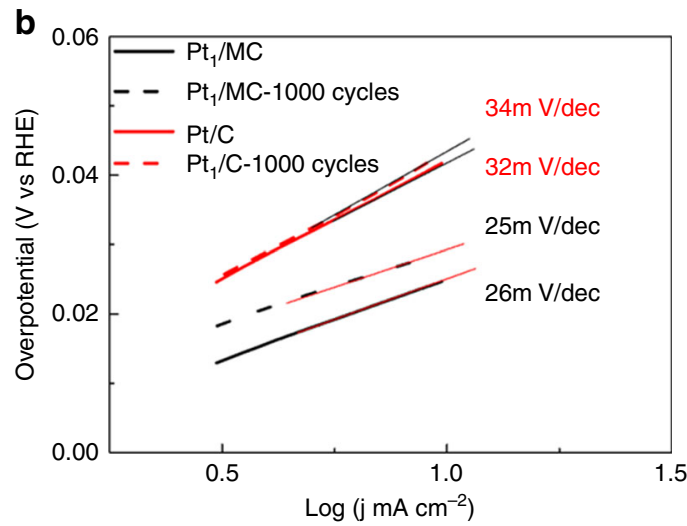

d

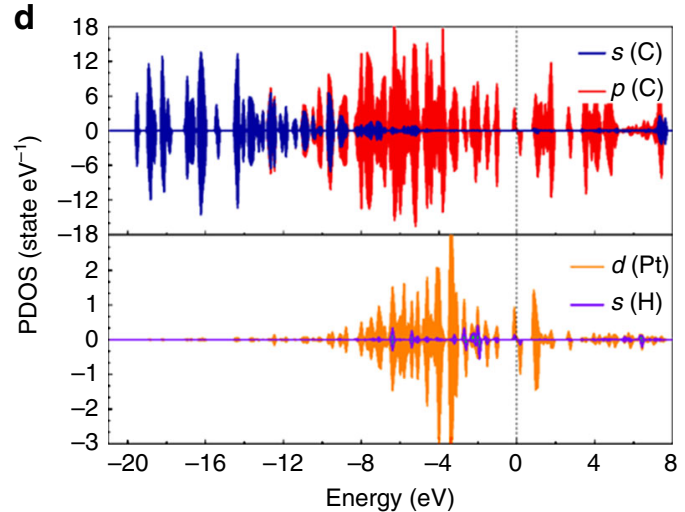

e

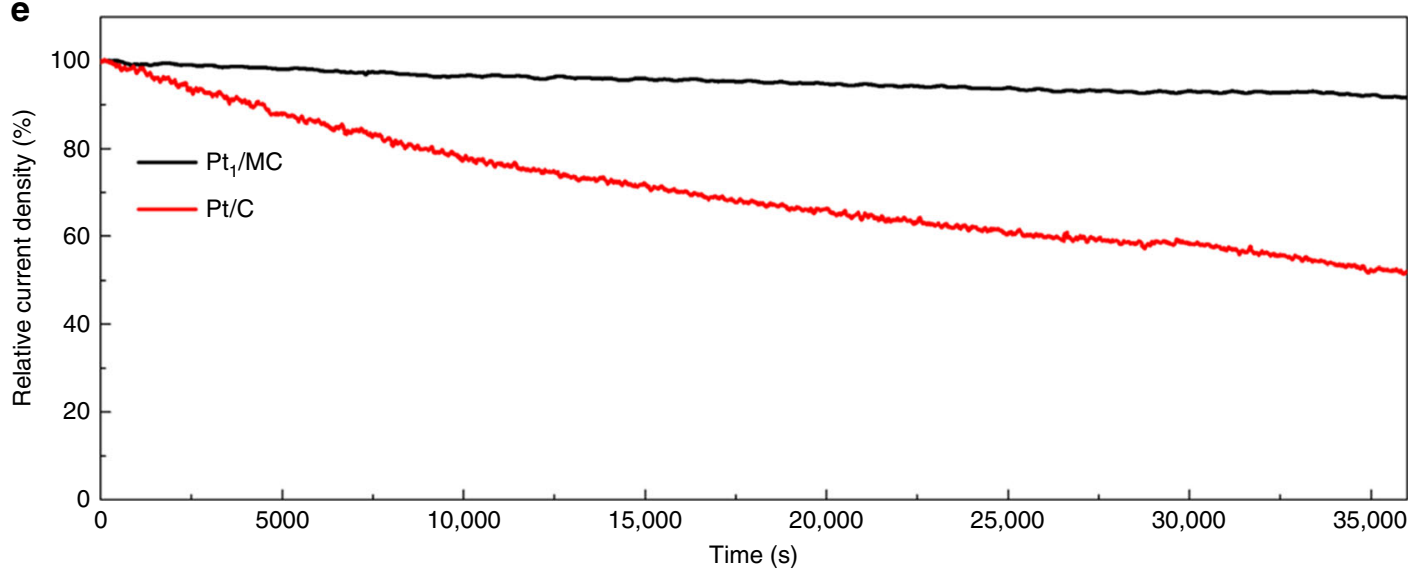

Fig. 4 HER activity of $\mathrm{Pt}_{1} / \mathrm{MC}$ and commercial $\mathrm{Pt} / \mathrm{C}$ in $0.5 \mathrm{M} \mathrm{H}_{2} \mathrm{SO}_{4}$. a Polarization curves of $\mathrm{Pt}_{1} / \mathrm{MC}$ and Pt/C before and after $1000 \mathrm{CV}$ cycles. b Tafel plots of $\mathrm{Pt}_{1} / \mathrm{MC}$ and $\mathrm{Pt} / \mathrm{C}$ (collected before and after $1000 \mathrm{CV}$ cycles). c Reaction mechanism for the HER, calculating $\Delta G_{H^{*}}$ for atomic $H$ adsorption on different defects/edge on mesoporous carbon. d Partial density of states (PDOS) of the Pt adsorbed on a single vacancy system after $\mathrm{H}$ adsorption. The Fermi level is shifted to zero. e Long-time running of $\mathrm{Pt}_{1} / \mathrm{MC}$ and $\mathrm{Pt} / \mathrm{C}$ electrocatalysts at the same overpotential of $30 \mathrm{mV}$

is difficult to determine by a computational method ${ }^{30}$. Here, only the relative oxidation state can be determined based on the Bader charge and $d$-orbital ${ }^{31}$. Considering that both $\mathrm{d}_{x y}$ and $\mathrm{d}_{x z}$ were partially occupied (not half-filled), the corresponding Pt state in $\mathrm{H}-\mathrm{Pt}-\mathrm{OH}$ was between approximately +1 and +2 . Correspondingly, the Bader charge results showed that one of the $\mathrm{H}$ in $\mathrm{H}-\mathrm{Pt}-$ $\mathrm{OH}$ changed to $-0.048 e$, which was reduced to the zero state.

Meanwhile, we initially started with the $\mathrm{Pt}(\mathrm{OH})_{2}{ }^{2-}$ in water system to check whether $\mathrm{Pt}(\mathrm{OH})_{2}$ instead of $\mathrm{H}-\mathrm{Pt}-\mathrm{OH}$ could be formed in the real situation during the fabrication of $\mathrm{Pt}$ single atoms. If $\mathrm{Pt}(\mathrm{OH})_{2}$ could be formed in the Pt-water system, the water should also be dissociated into hydroxyl and protons. Thus, the $\mathrm{Pt}(\mathrm{OH})_{2}$ was simulated along with two extra protons (Supplementary Fig. 12c). While the two hydrogen protons were initially located away from $\mathrm{Pt}(\mathrm{OH})_{2}$, the whole system remained electrically neutral. The $\mathrm{Pt}(\mathrm{OH})_{2}$ could spontaneously transform into $\mathrm{H}-\mathrm{Pt}-\mathrm{OH}$ along with the extra protons during $\mathrm{MD}$ simulations, in which one of the $\mathrm{OH}^{-}$in $\mathrm{Pt}(\mathrm{OH})_{2}$ reacted with $\mathrm{H}^{+}$to form $\mathrm{H}_{2} \mathrm{O}$, and the other hydrogen proton in the water bound to $\mathrm{Pt}$, as seen from the intermediate and final states in Supplementary Fig. 12c. This confirmed that H-Pt-OH was the stable species for $\mathrm{Pt}$ single atoms in aqueous solution instead of $\mathrm{Pt}$ $(\mathrm{OH})_{2}$. As for both $\mathrm{Pt}_{2}$ and $\mathrm{Pt}_{13}$, water dissociation also occurred on them, forming $\mathrm{H}-\mathrm{Pt}_{2}-\mathrm{OH}$ or $\mathrm{H}-\mathrm{Pt}_{13}-\mathrm{OH}$ like species, while either $\mathrm{Pt}_{2}$ or $\mathrm{Pt}_{13}$ itself remained intact and did not show any tendency to form any isolated $\mathrm{Pt}$, such as $\mathrm{H}-\mathrm{Pt}-\mathrm{OH}$. This result clearly indicated that it is vital to inhibit the formation of the $\mathrm{Pt}$ cluster at the initial step to retain the $\mathrm{Pt}$ in the form of single atoms, and no nucleation between two $\mathrm{H}-\mathrm{Pt}-\mathrm{OH}$ occurs in our 100 ps FPMD simulations. More interestingly, as shown in 
Supplementary Fig. 14, the $\mathrm{H}$ and $\mathrm{OH}$ in $\mathrm{H}-\mathrm{Pt}-\mathrm{OH}$ structure can be easily released as water on $\mathrm{MC}$, leaving the Pt single atom on MC with the release of approximately -0.345 and $-1.105 \mathrm{eV}$ at the single vacancy (SV) and double vacancy (DV) defect sites, respectively. This process further suggested that the $\mathrm{Pt}$ single atom in solution is an essential step for the fabrication of $\mathrm{Pt}_{1} / \mathrm{MC}$.

Another important question is whether the Pt single atoms can be stabilized on different surfaces. As mentioned above, atomically dispersed $\mathrm{Pt}$ on the surface of amorphous carbon, MC, MWCNTs, $\mathrm{TiO}_{2}$, and $\mathrm{ZnO}$ was successfully fabricated, while both Pt single atoms and clusters were found on graphene. For this purpose, the energy differences $\left(\Delta E_{c}\right)$ between the isolated $\mathrm{Pt}$ atom and the $\mathrm{Pt}$ dimer were calculated for graphene, $\mathrm{MC}$ and $\mathrm{TiO}_{2}$. Here, negative values mean clustering is most stable, and vice versa. First, considering the large negative value of $\Delta E_{c}$ $(-1.357 \mathrm{eV})$ for $\mathrm{Pt}_{1} /$ graphene, it was reasonable to expect the agglomeration of $\mathrm{Pt}$ single atoms on the graphene surface. Moreover, the graphene in our work was prepared by physical methods and contained few defective sites. There was almost no charge transfer occurring between $\mathrm{Pt}$ atoms and the perfect graphene to stabilize $\mathrm{Pt}$ atoms. Therefore, $\mathrm{Pt}$ atoms on perfect graphene were present as clusters and single atoms (Fig. 3e). Second, MC usually contains all kinds of defects, such as SV and DV, or edges. As shown in Supplementary Fig. 15, Supplementary Table 4, Pt single atoms preferred to adsorb on different defects, such as SVs, DVs or edges in MC. The isolated Pt atoms were more stable by at least $\sim 0.4 \mathrm{eV}$ than the corresponding Pt dimers located at one defect and an edge, as shown in Fig. 3d. Thus, Pt atoms energetically favoured the single-atom configuration rather than clustering on MC at defects/edges, while the Pt single atoms clustered on the pristine graphene, as observed in our STEM experiments (Fig. 2).

Next, let us discuss how the Pt single atoms anchor on the metal oxides. Here, we took anatase $\mathrm{TiO}_{2}$ as an example. It is wellknown that the oxygen vacancies in the anatase $\mathrm{TiO}_{2}(101)$ exist in the subsurface layer rather than on the surface ${ }^{32-34}$. Interestingly, our results showed that when the Pt single atoms adsorbed on the surface, the oxygen vacancy spontaneously migrated from the subsurface to the surface, leaving a vacancy site for Pt adsorption. The adsorption energy per Pt atom was $-4.10 \mathrm{eV}$ at the oxygen vacancy, which was smaller than the one $(-2.19 \mathrm{eV})$ on the perfect site of $\mathrm{TiO}_{2}$. Meanwhile, the isolated $\mathrm{Pt}$ atom on each vacancy was more stable by $0.91 \mathrm{eV}$ than the formation of one dimer on the vacancy (Supplementary Fig. 16). Thus, the Pt atoms preferred to form isolated atoms on each $\mathrm{O}_{v}$ rather than clustering on one $\mathrm{O}_{v}$ site of $\mathrm{TiO}_{2}$ (101). Furthermore, based on the successful synthesis of $\mathrm{Pt}$ single atoms, we proposed that the iced-photochemical method could be further developed as a more generalized strategy to fabricate other materials. We processed the $\mathrm{AgNO}_{3}$ and $\mathrm{H}_{2} \mathrm{AuCl}_{4}$ solution with a similar iced-photochemical reduction and obtained $\mathrm{Ag}$ and $\mathrm{Au}$ single atoms homogeneously dispersed on ultrathin carbon film (Fig. 2h, i, Supplementary Figs. 17-18).

Electrocatalyst performance. Downsizing $\mathrm{Pt}$ metals to single atoms certainly provided an effective approach to maximizing the atom efficiency for their catalytic applications ${ }^{1-6}$. Electrocatalysis of the hydrogen evolution reaction (HER), which has been widely used as a fundamental important process for water splitting and hydrogen production, was chosen as a model reaction. $\mathrm{A} \mathrm{Pt}_{1} / \mathrm{MC}$ (Supplementary Fig. 19) electrode was connected to a typical three-electrode setup in $0.5 \mathrm{M} \mathrm{H}_{2} \mathrm{SO}_{4}$ electrolyte at room temperature to evaluate the catalytic performance. Our results showed that the $\mathrm{Pt}_{1} / \mathrm{MC}$ catalysts exhibited a comparable HER activity to that of commercial $\mathrm{Pt} / \mathrm{C}$ catalysts at the same $\mathrm{Pt}$ loading of $10 \mu \mathrm{g} \mathrm{cm}^{-2}$ (Fig. 4a, Supplementary Fig. 20). The overpotential at a current density of $100 \mathrm{~mA} \mathrm{~cm}^{-2}$ (equal to a mass activity of $10 \mathrm{~A} \mathrm{mg}^{-1}$ ) for $\mathrm{Pt}_{1} / \mathrm{MC}$ was $65 \mathrm{mV}$ (Supplementary Fig. 21, Supplementary Fig. 22), with a promising decrease of more than $30 \mathrm{mV}$ compared to the commercially available $\mathrm{Pt} / \mathrm{C}$ catalyst as well as other single atom Pt-based catalysts (Supplementary Table 5). Importantly, $\mathrm{Pt}_{1} / \mathrm{MC}$ presented a negligible overpotential increase, while commercial $\mathrm{Pt} / \mathrm{C}$ catalysts showed a $22 \mathrm{mV}$ overpotential increase after 1000 cyclic voltammetry (CV) sweeps. Actually, we have measured the HER polarization curves for the reactivated $\mathrm{Pt} / \mathrm{C}$ sample after stability measurements for ten hours at an overpotential of $30 \mathrm{mV}$, which exhibited an enlarged overpotential at the current density of 100 $\mathrm{mA} \mathrm{cm}{ }^{-2}$ (Supplementary Fig. 23). This suggested that the deceased activity of $\mathrm{Pt} / \mathrm{C}$ can be attributed to irreversible detachment and/or agglomeration of the Pt nanoparticles in the $\mathrm{Pt} / \mathrm{C}$ sample, which is in line with previous reports ${ }^{35}, 36$. By contrast, $\mathrm{Pt}_{1} / \mathrm{MC}$ showed a negligible overpotential increase after 1000 cyclic voltammetry sweeps at the same conditions, attributed to the strong interaction between the isolated $\mathrm{Pt}$ atoms and MC. As shown in the STEM image of $\mathrm{Pt}_{1} / \mathrm{MC}$ after $1000 \mathrm{CV}$ cycles (Supplementary Fig. 24), almost no clusters can be observed with a similar density of Pt single atoms on mesoporous carbon. Moreover, the mass activity of $\mathrm{Pt}_{1} / \mathrm{MC}$ and $\mathrm{Pt} / \mathrm{C}$ is shown in Supplementary Fig. 25, in which the mass activity of $\mathrm{Pt}_{1} / \mathrm{MC}$ was more than three times greater relative to that of $\mathrm{Pt} / \mathrm{C}$ from 40 $\mathrm{mV}$ to $60 \mathrm{mV}$ overpotential, especially for the $\mathrm{Pt}_{1} / \mathrm{MC}$ sample after the stability measurement of $1000 \mathrm{CV}$ cycles. To provide further insight into the HER mechanisms on $\mathrm{Pt}_{1} / \mathrm{MC}$ and $\mathrm{Pt} / \mathrm{C}$ catalysts, Fig. $4 \mathrm{~b}$ shows that the Tafel plots of $\mathrm{Pt}_{1} / \mathrm{MC}$ and $\mathrm{Pt} / \mathrm{C}$ catalysts were $\sim 30 \mathrm{mV} \mathrm{dec}{ }^{-1}$, consistent with the previous reports and the known mechanism of the HER on Pt-based HER catalysts ${ }^{35}$. The relatively smaller Tafel slopes of $\mathrm{Pt}_{1} / \mathrm{MC}$, derived from both the initial HER polarization curve and the one after $1000 \mathrm{CV}$ cycles, strongly indicated the superior HER performances of $\mathrm{Pt}_{1} / \mathrm{MC}$. As discussed above, these results suggested the significantly enhanced HER specific activity of atomically dispersed platinum on mesoporous carbon at a lower consumption of precious $\mathrm{Pt}$. To further assess the long-term stability of $\mathrm{Pt}_{1} / \mathrm{MC}$ and $\mathrm{Pt} / \mathrm{C}$ catalysts as another important issue in the development of advanced electrocatalysts, chronopotentiometric measurements at a fixed overpotential of $30 \mathrm{mV}$ over extended periods as long as $10 \mathrm{~h}$ are presented in Fig. 4e, showing that there was a very small decay for $\mathrm{Pt}_{1} / \mathrm{MC}$ and an obvious decline for $\mathrm{Pt} / \mathrm{C}$ ( $8.4 \%$ vs. $48.0 \%)$. This sharp contrast indicated that the significant stability of $\mathrm{Pt}_{1} / \mathrm{MC}$ catalysts could be ascribed to a relatively stronger interaction between the Pt single atoms and mesoporous carbon substrates.

\section{Discussion}

It is important to understand why $\mathrm{Pt}_{1} / \mathrm{MC}$ exhibits a more active HER performance compared to commercial $\mathrm{Pt} / \mathrm{C}$ catalyst. For this purpose, the Gibbs free energies for atomic $\mathrm{H}$ adsorption, $\Delta G_{H^{*}}$, on different $\mathrm{MC}$ defects and edges were systematically considered and compared to pure Pt (111). As shown in Fig. 4c, Supplementary Fig. 26, the Pt single atom on MC can adsorb more than one hydrogen atom ${ }^{35}$; thus, the hydrogen production on Pt single atoms grown on MC can follow the Tafel mechanism $\left(2 \mathrm{H}^{*} \rightarrow \mathrm{H}_{2}\right)$. Interestingly, a smaller negative $\Delta G_{H^{*}}$ was observed for all $\mathrm{Pt}$ single atoms adsorbed on the MC with a $\mathrm{SV}\left(\mathrm{Pt}_{\mathrm{SV}}\right)$, a $\mathrm{DV}\left(\mathrm{Pt}_{\mathrm{DV}},\right)$ and an edge $\left(\mathrm{Pt}_{\mathrm{Edge}}\right)$. The calculated $\Delta G_{H^{*}}$ values for $\mathrm{H}$ adsorbed on $\mathrm{Pt}_{\mathrm{SV}}, \mathrm{Pt}_{\mathrm{DV}}$ and $\mathrm{Pt}_{\mathrm{Edge}}$ sites were $-0.049,0.080$, and $-0.075 \mathrm{eV}$, respectively. The $\Delta G_{H^{*}}$ for the Pt single atom systems became smaller than the corresponding value for commercial $\mathrm{Pt} / \mathrm{C}$, confirming the enhancement of HER activities in Pt single atoms loaded on MC. 
The HER activity mainly originates from the unique electronic properties of Pt single atoms on MC with respect to the conventional Pt surfaces. When Pt atoms are bound to MC, charge transfer occurs between $\mathrm{MC}$ and $\mathrm{Pt}^{37}$. For $\mathrm{Pt}_{1} / \mathrm{MC}$, the discrete 5d-orbitals of the Pt single atoms were mixed with the $\mathrm{C} 2 \mathrm{p}$ orbitals around the Fermi level for $\mathrm{Pt}_{\mathrm{Sv}}$ (Supplementary Fig. 28). The single Pt atom was positively charged $(+0.270 e)$, and the $\mathrm{C}$ atom obtained the electron based on Bader charge analysis (Supplementary Table 6). In this case, the Pt single atoms on the SV contained unoccupied 5d densities of states, which is vital for the $\mathrm{HER}^{38,39}$. Upon $\mathrm{H}$ chemisorption on $\mathrm{Pt}_{1} / \mathrm{MC}$ (Fig. $4 \mathrm{~d}$ ), the $5 \mathrm{~d}$ orbitals of the Pt single atoms interacted strongly with the $1 \mathrm{~s}$ orbital of the $\mathrm{H}$ atoms, leading to electron pairing and formation of hydride. In addition, more unoccupied $\mathrm{Pt}(5 \mathrm{~d})$ states were found above the Fermi level, which was consistent with the calculated charge transfer from the Pt atoms to the $\mathrm{H}$ atoms, as shown in Supplementary Table 6. This unique change in the electronic properties of the Pt single atoms on MC should be the primary reason for the increased HER activity of Pt single atoms on MC.

In conclusion, we have developed an iced-photochemical process to synthesize atomically dispersed $\mathrm{Pt}$ catalysts. Compared to commercial $\mathrm{Pt} / \mathrm{C}$ catalysts, the $\mathrm{Pt}$ single atoms loaded on mesoporous carbon achieved superior catalytic activity as well as improved stability for HER electrocatalysis. The iced-chemistry route not only provides a promising pathway for green synthesis of materials including single atoms and sub-nanometer clusters, but also opens up possibilities for tuning the solid nucleation and growth of wet-chemistry reactions. We propose that numerous conventional solution-phase chemical/photochemical reactions can use our iced-chemistry reactions to achieve the production of novel materials.

\section{Methods}

General information. Except when otherwise noted, all chemicals were purchased and used without purification. Additional details of materials and characterization techniques used are presented in Supplementary Methods.

Synthesis of $\mathbf{P t}_{1} / \mathbf{M C}$. A $0.3 \mathrm{mg} \mathrm{ml}^{-1} \mathrm{H}_{2} \mathrm{PtCl}_{6}$ solution was prepared using chloroplatinic acid and deionized water. A $1.5 \mathrm{ml} \mathrm{H}_{2} \mathrm{PtCl}_{6}$ solution was frozen quickly and entirely using liquid nitrogen. Then, the $\mathrm{H}_{2} \mathrm{PtCl}_{6}$ ice was irradiated by a UV lamp for $1 \mathrm{~h}$; meanwhile, the environment was kept at $-25^{\circ} \mathrm{C}$ in a lyophilizer (LGJ-10N), avoiding the melting of ice. The power density of the UV light near ice was measured to be $0.89 \mathrm{~mW} \mathrm{~cm}^{-2}$ using a radiometer (PL-MW 2000). After UV irradiation, the $\mathrm{H}_{2} \mathrm{PtCl}_{6}$ ice was melted at room temperature. All of these treatments were performed in a dark environment except for the UV irradiation treatment. Therefore, a Pt single atom solution was prepared. The mixed solution was composed of a $10 \mathrm{ml} 5 \mathrm{mg} \mathrm{ml}^{-1}$ mesoporous carbon solution and a $25 \mathrm{ml} 0.3$ $\mathrm{mg} \mathrm{ml}^{-1} \mathrm{Pt}$ single-atom solution and was filtered. The mesoporous carbon was synthesized using a facile aqueous route ${ }^{40,41}$. Then, the mixed samples were cleaned entirely using deionized water to remove the unreacted $\mathrm{H}_{2} \mathrm{PtCl}_{6}$. The $\mathrm{Pt}_{1} / \mathrm{MC}$ samples were dried naturally at room temperature.

XAFS experiment and data processing. XAFS measurements at the Pt $\mathrm{L}_{3}$-edge in both transmission (for Pt foil) and fluorescence (for samples) mode were performed at BL14W ${ }^{42}$ in the Shanghai Synchrotron Radiation Facility (SSRF). The electron beam energy was $3.5 \mathrm{GeV}$, and the stored current was $260 \mathrm{~mA}$ (top-up). A 38-pole wiggler with a maximum magnetic field of $1.2 \mathrm{~T}$ inserted in the straight section of the storage ring was used. XAFS data were collected using a fixed-exit double-crystal Si (111) monochromator. A Lytle detector was used to collect the fluorescence signal, and the energy was calibrated using a Pt foil. The photon flux at the sample position was $2.1 \times 10^{12}$ photons per second.

The raw data analysis was performed by using the IFEFFIT software package according to the standard data analysis procedures ${ }^{43}$. The spectra were calibrated and averaged, the pre-edge background was subtracted, and the spectra were postedge normalized using the Athena program in the IFEFFIT software package. The Fourier transformation of the $k^{3}$-weighted EXAFS oscillations, $k^{3} \cdot \chi(k)$, from $k$ space to $\mathrm{R}$ space was performed over the range of $3.0-11.5 \AA^{-1}$ (3.0-14.2 for Pt foil) to obtain a radial distribution function. Data fitting was performed by the Artemis program in IFEFFIT.

Electrocatalytic measurements. The catalyst dispersion or ink was prepared by mixing commercial Pt/C (20 wt\%) or as-prepared $\mathrm{Pt}_{1} / \mathrm{MC}$ (2.6 wt\%, confirmed by an ICP-MS test) in a $2 \mathrm{ml}$ mixture of $1.0 \mathrm{ml}$ isopropyl alcohol, $0.95 \mathrm{ml}$ water and a $0.05 \mathrm{ml} 5 \mathrm{wt} \%$ Nafion solution followed by ultrasonication for $30 \mathrm{~min}$. For Pt/C, after dispersing $2 \mathrm{mg}$ of commercial $\mathrm{Pt} / \mathrm{C}$ in $2 \mathrm{ml}$ of mixture solution, the concentration of Pt element in $\mathrm{Pt} / \mathrm{C}$ was $0.2 \mathrm{mg} \mathrm{m}^{-1}$. Then, $9.8 \mathrm{ml}$ of the ink was uniformly loaded onto the freshly polished glassy carbon electrodes (diameter $=5$ $\mathrm{mm}$ ), and the corresponding loading of Pt amounts was $0.01 \mathrm{mg} \mathrm{cm}^{-2}$ $\left(10 \mu \mathrm{g} \mathrm{cm}^{-2}\right)$. For $\mathrm{Pt}_{1} / \mathrm{MC}, 37.7 \mathrm{ml}$ of the ink $\left(0.052 \mathrm{mg} \mathrm{ml}^{-1} \mathrm{Pt}\right.$ element concentration after $4 \mathrm{mg} \mathrm{Pt} / \mathrm{MC}$ was dispersed in $2 \mathrm{ml}$ mixture solution) was also uniformly loaded onto the same freshly polished glassy carbon electrodes, and the corresponding loading of Pt amounts was also $0.01 \mathrm{mg} \mathrm{cm}^{-2}$.

All electrochemical tests were performed in a conventional three-electrode electrochemical cell using an Autolab potentiostat (PGSTAT-302N) equipped with the Nova 1.11 software. A rotating-disk glassy-carbon electrode or an L-form glassy-carbon electrode was used as the working electrode, while a carbon rod electrode and $\mathrm{Ag} / \mathrm{AgCl}$ (with saturated $\mathrm{KCl}$ as the filling solution) were used as the counter and reference electrodes, respectively. All potentials measured were calibrated to the reversible hydrogen electrode (RHE) using the following equation: $E_{\mathrm{RHE}}=E_{\mathrm{Ag} / \mathrm{AgCl}}+0.197+0.059^{*} \mathrm{pH}$. To accelerate the diffusion of the $\mathrm{H}_{2}$ gas bubbles formed at the catalyst surface during the hydrogen evolution reaction (HER), the polarization curves were recorded using a rotating-disk electrode with $90 \%$ IR compensation at the rotation rate of 1600 r.p.m. A total of 1000 cyclic voltammetry sweeps were carried out at the voltage range from +0.3 to $-0.1 \mathrm{~V}$ (vs. RHE) with a scan rate of $100 \mathrm{mV} \mathrm{s}^{-1}$ in $0.5 \mathrm{M} \mathrm{H}_{2} \mathrm{SO}_{4}$ to test the stability of $\mathrm{Pt}_{1} / \mathrm{MC}$ and $\mathrm{Pt} / \mathrm{C}$ catalysts; meanwhile the stability measurements were also conducted using an L-form electrode at the overpotential of $30 \mathrm{mV}$.

DFT calculations. Our calculations were performed based on density functional theory (DFT) calculations, as implemented in the Vienna ab initio package $(\text { VASP })^{44,45}$. The general gradient approximation of Perdew-Burke-Ernzerhof (GGA-PBE) was adopted for the exchange-correlation functional ${ }^{46}$. Moreover, the electron wave functions were expanded with a plane wave cutoff of $400 \mathrm{eV}$. The $(10 \times 10 \times 1)$ supercell containing 200 carbon atoms was constructed using periodic boundary conditions (PBCs), and the vacuum layers were set to be larger than $20 \AA$ to avoid periodic interactions. Reciprocal space integration was performed by the Monkhorst-Pack special k-point scheme with $2 \times 2 \times 1$ grid meshes for the structure relaxation for the $\mathrm{Pt}$ adsorption mesoporous carbon (MC). Atomic relaxation was performed until the total energy variation was less than $10^{-6} \mathrm{eV}$ and all forces on each atom were less than $0.01 \mathrm{eV} \AA^{-1}$. The DFT-D3 approach was used to evaluate the effect of the van der Waals (vdW) interaction ${ }^{47}$. Bader charge ana$\mathrm{lysis}^{48}$ was performed to quantitatively estimate the amount of charge transfer between the adsorbed $\mathrm{Pt}$ (or $\mathrm{H}$ ) and the mesoporous carbon. To investigate whether Pt will cluster on the anatase (101) surface, the adsorption energies of $\mathrm{Pt}$ on a $(1 \times 4)$ repeated surface supercell $(10.35 \AA \times 15.21 \AA)$ were calculated. Oxygen vacancies $\left(\mathrm{O}_{\mathrm{v}}\right)$ generally exist in anatase $\mathrm{TiO}_{2}$, and all the models used in the calculations contained an $\mathrm{O}_{\mathrm{v}}$ defect.

The first-principles molecular dynamics (FPMD) calculations were performed using the CP2K/QUICKSTEP package ${ }^{49}$. The wave functions of the valence electrons were expanded in terms of Gaussian functions with molecularly optimized double- $\zeta$ polarized basis sets (m-DZVP), ensuring a small basis set superposition error ${ }^{50}$, and core electrons were described with norm-conserving Goedecker, Teter, and Hutter (GTH) pseudopotentials ${ }^{51}$. The FPMD was carried out with the NVT ensemble at the target temperature of $300 \mathrm{~K}$ or $1000 \mathrm{~K}$ using the Nose-Hoover thermostat. The time step in all calculations was $1 \mathrm{fs}$, and each sample was equilibrated at the target temperature for $50 \mathrm{ps}$, which is considered long enough for nucleation or reactions on MC or metal oxides.

Data availability. All data are available from the authors upon reasonable request.

Received: 21 March 2017 Accepted: 22 September 2017 Published online: 14 November 2017

\section{References}

1. Liu, P. et al. Photochemical route for synthesizing atomically dispersed palladium catalysts. Science 352, 797-800 (2016).

2. Guo, X. et al. Direct, nonoxidative conversion of methane to ethylene, aromatics, and hydrogen. Science 344, 616-619 (2014).

3. Jones, J. et al. Thermally stable single-atom platinum-on-ceria catalysts via atom trapping. Science 353, 150-154 (2016).

4. Ding, K. et al. Identification of active sites in $\mathrm{CO}$ oxidation and water-gas shift over supported Pt catalysts. Science 350, 189-192 (2015).

5. Qiao, B. et al. Single-atom catalysis of $\mathrm{CO}$ oxidation using $\mathrm{Pt}_{1} / \mathrm{FeOx}$. Nat. Chem. 3, 634-641 (2011)

6. $\mathrm{Li}, \mathrm{X}$. et al. Single-atom Pt as Co-Catalyst for enhanced photocatalytic $\mathrm{H}_{2}$ evolution. Adv. Mater. 28, 2427-2431 (2016). 
7. Tan, S. F., Lin, G., Bosman, M., Mirsaidov, U. \& Nijhuis, C. A. Real-time dynamics of galvanic replacement reactions of silver nanocubes and Au studied by liquid-cell transmission electron microscopy. ACS Nano 10, 7689-7695 (2016).

8. Cheng, N. et al. Platinum single-atom and cluster catalysis of the hydrogen evolution reaction. Nat. Commun. 7, 13638 (2016).

9. Wang, H. et al. Direct and continuous strain control of catalysts with tunable battery electrode materials. Science 354, 1031-1036 (2016).

10. Woods, J. M. et al. One-step synthesis of $\mathrm{MoS}_{2} / \mathrm{WS}_{2}$ layered heterostructures and catalytic activity of defective transition metal dichalcogenide films. ACS Nano 10, 2004-2009 (2016).

11. $\mathrm{Bu}, \mathrm{L}$. et al. Biaxially strained $\mathrm{PtPb} / \mathrm{Pt}$ core/shell nanoplate boosts oxygen reduction catalysis. Science 354, 1410-1414 (2016).

12. Chen, G. et al. Interfacial effects in iron-nickel hydroxide-platinum nanoparticles enhance catalytic oxidation. Science 344, 495-499 (2014).

13. Buck, M. R., Bondi, J. F. \& Schaak, R. E. A total-synthesis framework for the construction of high-order colloidal hybrid nanoparticles. Nat. Chem. 4, 37-44 (2012).

14. Li, M. et al. Ultrafine jagged platinum nanowires enable ultrahigh mass activity for the oxygen reduction reaction. Science 354, 1414-1419 (2016).

15. Xia, Y., Gilroy, K. D., Peng, H. C. \& Xia, X. Ultrathin metal-organic framework nanosheets for electrocatalytic oxygen evolution. Angew. Chem. Int. Ed. 56 60-95 (2017).

16. Wang, X., Zhuang, J., Peng, Q. \& Li, Y. A general stratege for nanocrystal synthesis. Nature 437, 121-124 (2005).

17. Loh, N. D. et al. Multistep nucleation of nanocrystals in aqueous solution. Nat. Chem. 9, 77-82 (2017).

18. Lu, J., Aydin, C., Browning, N. D. \& Gates., B. C. A single-site platinum CO oxidation catalyst in Zeolite KLTL: microscopic and spectroscopic determination of the locations of the platinum atoms. Angew. Chem. Int. Ed. 51, 5842-5846 (2012).

19. Qiu, H. J. et al. Nanoporous graphene with single-atom nickel dopants: An efficient and stable catalyst for electrochemical hydrogen production. Angew. Chem. Int. Ed. 54, 14031-14035 (2015).

20. Choi, C. H. et al. Tuning selectivity of electrochemical reactions by atomically dispersed platinum catalyst. Nat. Commun. 7, 10922 (2016).

21. Yin, P. et al. Single cobalt atoms with precise N-coordination as superior oxygen reduction reaction catalysts. Angew. Chem. Int. Ed. 55, 10800-10805 (2016).

22. Liu, L. et al. Generation of subnanometric platinum with high stability during transformation of a 2D zeolite into 3D. Nat. Mater. 16, 132-138 (2016).

23. Kim, Y. et al. Flexible metal-oxide devices made by room-temperature photochemical activation of sol-gel films. Nature 489, 128-133 (2012).

24. Kärkäs, M. D., Porco, J. A. \& Stephenson, C. R. J. Photochemical approaches to complex chemotypes: applications in natural product synthesis. Chem. Rev. 116, 9683-9747 (2016).

25. Huo, H. et al. Asymmetric photoredox transition-metal catalysis activated by visible light. Nature 515, 100-103 (2014).

26. Linic, S., Aslam, U., Boerigter, C. \& Morabito, M. Photochemical transformations on plasmonic metal nanoparticles. Nat. Mater. 14, 567-576 (2015).

27. Zhai, Y. et al. Polyvinylpyrrolidone-induced anisotropic growth of gold nanoprisms in plasmon-driven synthesis. Nat. Mater. 15, 889-895 (2016).

28. Gu, H., Yang, Y., Tian, J. \& Shi, G. Photochemical synthesis of noble metal (Ag, $\mathrm{Pd}, \mathrm{Au}, \mathrm{Pt}$ ) on graphene/ $\mathrm{ZnO}$ multihybrid nanoarchitectures as electrocatalysis for $\mathrm{H}_{2} \mathrm{O}_{2}$ reduction. ACS Appl. Mater. Interfaces 5, 6762-6768 (2013).

29. Emmrich, M. et al. Subatomic resolution force microscopy reveals internal structure and adsorption sites of small iron clusters. Science 348, 308-311 (2015).

30. Raebiger, H. et al. Charge self-regulation upon changing the oxidation state of transition metals in insulators. Nature 453, 763-766 (2008).

31. Sit, P. H. L. et al. Unambiguous theoretical approach to oxidation state determination via first-principles calculations. Inorg. Chem. 50, 10259-10267 (2011).

32. Cheng, H. \& Selloni, A. Surface and subsurface oxygen vacancies in anatase $\mathrm{TiO}_{2}$, and differences with rutile. Phys. Rev. B 79, 2101 (2009).

33. Cuan, Q., Tao, J., Gong, X.-Q. \& Batzill, M. Adsorbate induced restructuring of $\mathrm{TiO}_{2}(011)-(2 \times 1)$ leads to one-dimensional nanocluster formation. Phys. Rev. Lett. 108, 106105 (2012)

34. Nørskov, J. K., Bligaard, T., Rossmeisl, J. \& Christensen, C. H. Towards the computational design of solid catalysts. Nat. Chem. 1, 37-46 (2009).

35. Cheng, N. et al. Platinum single-atom and cluster catalysis of the hydrogen evolution reaction. Nat. Commun. 7, 13638 (2016).

36. Meier, J. C. et al. Stability investigations of electrocatalysts on the nanoscale. Energy Environ. Sci. 5, 9319-9330 (2012).

37. Nørskov, J. K. et al. Trends in the exchange current for hydrogen evolution. J. Electrochem. Soc. 152, J23-J26 (2005).

38. Ghosh, T. K. \& Nair, N. N. $\mathrm{Rh}_{1} / \gamma-\mathrm{Al}_{2} \mathrm{O}_{3}$ single-atom catalysis of $\mathrm{O}_{2}$ zctivation and $\mathrm{CO}$ oxidation: Mechanism, effects of hydration, oxidation state, and cluster size. ChemCatChem 5, 1811-1821 (2013).
39. Zhang, X., Shi, H. \& Xu, B. Catalysis by gold: Isolated surface $\mathrm{Au}^{3+}$ ions are active sites for selective hydrogenation of 1,3-Butadiene over $\mathrm{Au} / \mathrm{ZrO}_{2}$ Catalysts. Angew. Chem. Int. Ed. 44, 7294-7297 (2005).

40. Liu, D. et al. Highly efficient synthesis of ordered nitrogen-doped mesoporous carbons with tunable properties and its application in high performance supercapacitors. J. Power Sources 321, 143-154 (2016).

41. Liu, D. et al. One-pot aqueous route to synthesize highly ordered cubic and hexagonal mesoporous carbons from resorcinol and hexamine. Carbon N. Y. 50, 476-487 (2012).

42. Yu, H. et al. The XAFS beamline of SSRF. Nucl. Sci. Tech. 26, 050102 (2015)

43. Newville, M. IFEFFIT: interactive XAFS analysis and FEFF fitting. J. Synchrotron Rad. 8, 322-324 (2001).

44. Kresse, G. \& Furthmüller, J. Efficiency of ab-initio total energy calculations for metals and semiconductors using a plane-wave basis set. Comput. Mater. Sci. 6 , 15-50 (1996)

45. Kresse, G. \& Furthmüller, J. Efficient iterative schemes for ab initio total-energy calculations using a plane-wave basis set. Phys. Rev. B 54, 11169 (1996).

46. Perdew, J. et al. Atoms, molecules, solids, and surfaces: Applications of the generalized gradient approximation for exchange and correlation. Phys. Rev. B 46, 6671 (1992).

47. Grimme, S., Antony, J., Ehrlich, S. \& Krieg, H. A consistent and accurate ab initio parametrization of density functional dispersion correction (DFT-D) for the 94 elements H-Pu. J. Chem. Phys. 132, 154104 (2010).

48. Henkelman, G., Arnaldsson, A. \& Jónsson, H. A fast and robust algorithm for Bader decomposition of charge density. Comp. Mater. Sci. 36, 354-360 (2006).

49. VandeVondele, J. et al. Quickstep: fast and accurate density functional calculations using a mixed Gaussian and plane waves approach. Comput. Phys Commum. 167, 103-128 (2005).

50. VandeVondele, J. \& Hutter, J. Gaussian basis sets for accurate calculations on molecular systems in gas and condensed phases. J. Chem. Phys. 127, 114105 (2007).

51. Goedecker, S., Teter, M. \& Hutter, J. Separable dual-space Gaussian pseudopotentials. Phys. Rev. B Condens. Matter. 54, 1703-1710 (1996).

\section{Acknowledgements}

We acknowledge the financial support from the National Basic Research of China (Grant Nos. 2015CB932500), the National Natural Science Foundation of China (NSFC) (Grant No. 51522207, 51661135025, 11374332, 51572016, U1530401, and 11422218), the National Program for Thousand Young Talents of China and the China Postdoctoral Science Foundation (Grant No. 2016M601019). We thank the XAFS station (BL14W1) of the Shanghai Synchrotron Radiation Facility and computation supports from Tianhe-JK at the Beijing Computational Science Research Center (CSRC).

\section{Author contributions}

H.W. conceived the research. H.H.W., K.H. and R.Z. prepared the sample. H.H.W., K.H., R.Z., B.G., J.M., S.Z., Q.L., M.L. and C.Z. performed the experiment. D.W., B.W. and L.-M.L. performed simulations. H.H.W., K.H., B.G., L.-M.L. and H.W. prepared the manuscript with extensive suggestions and contributions from all the other authors.

\section{Additional information}

Supplementary Information accompanies this paper at doi:10.1038/s41467-017-01521-4

Competing interests: The authors declare no competing financial interests.

Reprints and permission information is available online at http://npg.nature.com/ reprintsandpermissions/

Publisher's note: Springer Nature remains neutral with regard to jurisdictional claims in published maps and institutional affiliations.

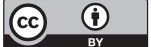

Open Access This article is licensed under a Creative Commons Attribution 4.0 International License, which permits use, sharing, adaptation, distribution and reproduction in any medium or format, as long as you give appropriate credit to the original author(s) and the source, provide a link to the Creative Commons license, and indicate if changes were made. The images or other third party material in this article are included in the article's Creative Commons license, unless indicated otherwise in a credit line to the material. If material is not included in the article's Creative Commons license and your intended use is not permitted by statutory regulation or exceeds the permitted use, you will need to obtain permission directly from the copyright holder. To view a copy of this license, visit http://creativecommons.org/ licenses/by/4.0/

(C) The Author(s) 2017 